\title{
Evaluación de la comunidad desnitrificante tipo nosZ en la columna de agua de Isla del Sol, embalse de Prado, Tolima
}

\author{
Maribeb Castro-González \\ Departamento de Biología, Facultad de Ciencias, Universidad del Tolima
}

\begin{abstract}
Resumen
En este trabajo se evaluó la diversidad genética de las comunidades desnitrificantes relacionadas con el reciclaje del gas invernadero óxido nitroso $\left(\mathrm{N}_{2} \mathrm{O}\right)$ en la columna de agua de Isla del Sol, en el embalse de Prado, mediante el análisis del polimorfismo de los fragmentos de restricción terminal del gen funcional nosZ, que codifica para la reducción de $\mathrm{N}_{2} \mathrm{O}$ a $\mathrm{N}_{2}$ durante la desnitrificación. Este es el primer reporte que demuestra la presencia de microorganismos desnitrificantes tipo nosZ en un ecosistema acuático colombiano. Los resultados indicaron que la digestión con la enzima MspI generó un mejor perfil de la comunidad nosZ (expresado en una mayor riqueza de fragmentos de restricción terminal, FRT), que el obtenido con la enzima HhaI. Además, la comunidad de tipo nosZ presentó mayor riqueza y diversidad (índice de Shannon $\left.-\mathrm{H}^{\prime}=0,9\right)$ de FRT en la profundidad (9m) a la cual se encontraron condiciones subóxicas $\left(1,1 \mathrm{mg} / 1 \mathrm{de}_{2}\right)$ y altos niveles de nitrato $(0,78 \mathrm{mg} / \mathrm{l})$, lo cual podría favorecer la reducción de $\mathrm{N}_{2} \mathrm{O}$ a $\mathrm{N}_{2}$. Estos resultados son la base para el desarrollo de investigaciones futuras que permitan establecer la funcionalidad y significancia de estas comunidades en la regulación de las emisiones de $\mathrm{N}_{2} \mathrm{O}$ desde esta hidroeléctrica.
\end{abstract}

Palabras clave: desnitrificación, embalse, gen funcional, suboxia, hipolimnion.

Evaluation of the nosZ-type denitrifier community in Isla del Sol water column, Prado reservoir, Tolima

\begin{abstract}
In the present study the genetic diversity of denitrifying communities related to the recycling of greenhouse gas nitrous oxide $\left(\mathrm{N}_{2} \mathrm{O}\right)$ in Isla del Sol water column, Prado dam (Tolima), was analyzed using the terminal restriction fragment lenght polymorphism analysis of the nosZ functional gene, which encodes the $\mathrm{N}_{2} \mathrm{O}$ reduction to $\mathrm{N}_{2}$ during denitrification. This is the first report demonstrating the presence of nosZ-type denitrifying microorganisms in a Colombian acquatic ecosystem. The results indicated that digestion with the MspI enzyme generated a better nosZ community profile (expresed in an increase of terminal restriction fragments - TRF) than the HhaI enzyme. Besides, the nosZ community had a higher TRF richness and diversity (Shannon index $-H^{\prime}=0.9$ ) at the depth $(9 \mathrm{~m}$ ) where suboxic conditions $\left(1.1 \mathrm{mg} / \mathrm{L} \mathrm{O}_{2}\right)$, as well as high nitrate levels $(0.78 \mathrm{mg} / \mathrm{L})$ were found, which could favoured $\mathrm{N}_{2} \mathrm{O}$ reduction to $\mathrm{N}_{2}$. These results are the base for future studies on the functionality and significance of such communities for the regulation of $\mathrm{N}_{2} \mathrm{O}$ emissions from this dam.
\end{abstract}

Key words: Denitrification, reservoir, functional genes, suboxia, hypolimnion.

\section{Introducción}

La desnitrificación (canónica y quimiolitotrófica) y la oxidación de amonio anaeróbico (anaerobic ammonium oxidation, anammox) son los principales procesos microbianos que producen $\mathrm{N}_{2}$ a partir de la remoción del nitrógeno fijado en diversos hábitats acuáticos, incluidos los lagos (Hulth, et al., 2005, Schubert, et al., 2006, Harrison, et al., 2009, Bai, et al., 2012, Wen, et al., 2012, Wenk, et al., 2013) y los embalses (Deemer, et al., 2011; Beaulieu, et al., 2014). Sin embargo, solo la desnitrificación puede generar $\mathrm{N}_{2} \mathrm{O}$ durante la reducción del nitrato a gas dinitrógeno (Zumft \& Kroneck,
2007) con niveles bajos de $\mathrm{O}_{2}$. Existe una amplia variedad de grupos taxonómicos en los dominios de Bacteria y Archaea (Zumft, 1997) que llevan a cabo este proceso. La reducción de nitrito a óxido nítrico distingue las bacterias desnitrificantes de otras que respiran nitrato. Esta reacción es catalizada por dos reductasas de nitrito diferentes: una citocromo $\mathrm{Cd}^{-1}$ codificada por el gen nirS, y una enzima que contiene cobre codificada

\section{Correspondencia:}

Maribeb Castro-González, mcastro@ut.edu.co

Recibido: 10 de octubre de 2014

Aceptado: 2 de diciembre de 2014 
por el gen nirK. La reducción de óxido nitroso $\left(\mathrm{N}_{2} \mathrm{O}\right)$, último paso de la desnitrificación, es catalizada por la reductasa de óxido nitroso codificada por el gen nosZ. Esta enzima se encuentra en todas las bacterias desnitrificantes que reducen nitrato a dinitrógeno y también en unas pocas bacterias no desnitrificantes como Wolinella (Vibrio) succinogenes, que usa el $\mathrm{N}_{2} \mathrm{O}$ como un aceptor terminal de electrones (Zumft, 1997, Zumft \& Kroneck, 2007).

En condiciones naturales, y dependiendo del nivel de $\mathrm{O}_{2}$ imperante, la columna de agua puede presentar hipoxia $\left(\leq 1,4 \mathrm{ml} / 1 \mathrm{O}_{2}\right)$, suboxia $\left(\leq 0,1 \mathrm{ml} / 1 \mathrm{O}_{2}\right)$, o anoxia $\left(0 \mathrm{ml} / 1 \mathrm{O}_{2}\right)$ (Naqvi, et al., 2010), lo que incide en diferentes aspectos de la desnitrificación como el incremento en la abundancia de bacterias desnitrificantes productoras de $\mathrm{N}_{2} \mathrm{O}$ en la interfase óxica-anóxica; el consumo de $\mathrm{N}_{2} \mathrm{O}$ por desnitrificación en aguas subóxicas y anóxicas; la producción de $\mathrm{N}_{2} \mathrm{O}$ por desnitrificación o la oxidación de $\mathrm{NH}_{4}^{+}$en aguas hipóxicas y óxicas de embalses (Deemer, et al., 2011; Beaulieu, et al., 2014), y la desnitrificación en microambientes anóxicos en aguas oxigenadas (Brettar \& Höfle, 1993; Naqvi, et al., 2000). Además, en varios estudios se ha analizado la composición de la comunidad desnitrificante en relación con la variación del oxígeno, el nitrato, el nitrito, los gradientes redox y la disponibilidad de materia orgánica (Castro-González, et al., 2005; Hallin, et al., 2007; Baxter, et al., 2012, Baxter, et al., 2013), considerándolos como factores clave en la regulación de la desnitrificación y del reciclaje de $\mathrm{N}_{2} \mathrm{O}$ en ecosistemas acuáticos y terrestres (Zumft, 1997).

Los lagos, embalses y ríos son sitios de desnitrificación activos y contribuyen en $\sim 20 \%$ a la remoción del nitrógeno global (Seitzinger, et al., 2006). Algunos modelos indican que los embalses o reservorios contribuyen más a la remoción de nitrógeno que los lagos (Harrison, et al., 2009), y que tienen gran importancia en el balance global del $\mathrm{N}_{2} \mathrm{O}$ (St. Louis, et al., 2000). Desde 1990 se sabe que los lagos artificiales y embalses hidroeléctricos emiten gases de efecto invernadero (Rudd, et al., 1993), y actualmente se estima que contribuyen con cerca del $7 \%$ a las emisiones originadas por la acción del hombre en áreas tropicales (St. Louis, et al., 2000). Aunque se han hecho estudios en algunos embalses sobre la producción y consumo de $\mathrm{N}_{2} \mathrm{O}$ por desnitrificación (Deemer, et al., 2011; Beaulieu, et al., 2014) y sobre los flujos de $\mathrm{N}_{2} \mathrm{O}$ en hidroeléctricas, principalmente en Brasil (Goldenfum, 2012), muy poco se conoce sobre la diversidad genética de las comunidades microbianas desnitrificantes de tipo nos $Z$ encargadas de su reducción; de ahí la importancia de iniciar estudios en esta área.

El estudio de la diversidad de las comunidades desnitrificantes en diversos ecosistemas acuáticos ha implicado el uso y el desarrollo de la genómica funcional, ya que estas constituyen un grupo polifilético (Hallin, et al., 2007). Los genes que codifican para la reductasa de nitrito (nirS y nirK) y la reductasa de óxido nitroso (nosZ) han permitido el análisis de la diversidad de las bacterias (Jones, et al., 2013) y las arqueobacterias desnitrificantes (Rusch, 2013) en ecosistemas acuáticos mediante el análisis de los fragmentos de restricción terminal y la secuenciación convencional de clones (Castro-González, et al., 2005;), la electroforesis en gel con gradiente de desnaturalización (denaturing gradient gel electrophoresis, DGGE) (Throbäck, et al., 2004), la reacción en cadena de la polimerasa cuantitativa (Dong, et al., 2009) y, más recientemente, la pirosecuenciación (Palmer, et al., 2012, Bowen, et al., 2013). La técnica de análisis de los fragmentos de restricción terminal se basa en la variación de la posición del sitio de restricción entre secuencias de una comunidad bacteriana, la cual genera diferentes fragmentos de restricción terminal (FRT) que son detectados por la emisión de fluorescencia en secuenciadores automáticos durante la electroforesis en gel de alta resolución. Esta técnica tiene ventajas frente a los demás métodos por su facilidad de manejo en el laboratorio y porque genera datos cuantitativos y precisos (tamaño en pares de bases e intensidad de la fluorescencia de cada FRT), lo que permite la diferenciación de comunidades microbianas en diversos ambientes con base en la riqueza y la abundancia relativa de los FRT estimados en el análisis (Dumbar, et al., 2001).

Teniendo en cuenta que el embalse de Prado en el sur del Tolima se caracteriza por ser un embalse eutrofizado, estratificado térmica y químicamente, con bajos niveles de oxígeno y gran cantidad de materia orgánica (Márquez \& Guillot, 1988), es probable que allí ocurran diversos procesos microbianos, entre ellos, la desnitrificación. Sin embargo, aunque la represa es considerada como una de las más grandes e importantes de Colombia, solo recientemente se viene abordando el estudio de la composición de las comunidades desnitrificantes, los ciclos biogeoquímicos y la producción de gases invernadero como el $\mathrm{N}_{2} \mathrm{O}$ en ella. Con respecto a este último aspecto, en algunos estudios recientes se han encontrado niveles elevados de $\mathrm{N}_{2} \mathrm{O}(36,5-46,3 \mu \mathrm{M})$ en la columna de agua de Isla del Sol (Castro-González \& Torres-Valdés, 2014), lo que ha generado inquietudes sobre si hay comunidades microbianas portadoras del gen nos $Z$ con potencial para reducir el $\mathrm{N}_{2} \mathrm{O}$ a $\mathrm{N}_{2}$ que ayuden a disminuir las eventuales emisiones de este gas invernadero desde el embalse hacia la atmósfera. Por lo anterior, los objetivos de esta investigación fueron comparar la composición de la comunidad desnitrificante de tipo nos $Z$ en aguas superficiales $(2 \mathrm{~m})$ y de fondo $(9 \mathrm{~m})$ en el área de Isla del Sol, y analizar las diferencias en la estructura de dicha comunidad con relación a los parámetros ambientales prevalentes.

\section{Metodología}

El muestreo se realizó en septiembre de 2012 en el embalse de Prado, ubicado en el sur del Tolima, a 365 msnm, específicamente en el área de Isla del Sol, localizada a $03^{\circ} 45^{\prime} \mathrm{N}$ y 
$04^{\circ} 51^{\prime} \mathrm{O}$. En este punto se determinó previamente el perfil vertical de oxígeno, la temperatura y el $\mathrm{pH}$ entre 0 y $10 \mathrm{~m}$ de profundidad con un equipo multiparámetro Oakton $\AA$, con el fin de establecer a qué profundidad se tomarían las muestras para el análisis molecular y de nutrientes. Con base en estos parámetros se tomaron las muestras de agua (5 litros) con botella vertical a 2 y 9 metros de profundidad, dado que entre esos dos niveles se observó contraste en la concentración de $\mathrm{O}_{2}$. A continuación se filtraron $100 \mathrm{ml}$ de agua en membranas con poros de $0,22 \mu \mathrm{m}$ de tamaño y se congelaron a $-20{ }^{\circ} \mathrm{C}$ para el posterior análisis de nitrógeno amoniacal mediante el método de Kjeldahl, y de nitrato y nitrito a $220-275 \mathrm{~nm}$ y 510 $\mathrm{nm}$, respectivamente, mediante técnicas espectrofotométricas y siguiendo los métodos descritos en el Standard Methods for the Examination of Water and Wastewater (1999) para análisis de nutrientes en aguas, todo lo cual se hizo en el correspondiente laboratorio de la Universidad del Tolima. El agua restante se refrigeró y se guardó en la oscuridad hasta su filtración consecutiva en membranas con poros de 20,8 y $0,22 \mu \mathrm{m}$ de tamaño. Las membranas de $0,22 \mu \mathrm{m}$ se congelaron en viales de congelación con tampón de lisis (400 $\mathrm{Mm} \mathrm{NaCl}, 0,75 \mathrm{M}$ sucrosa, $50 \mathrm{Mm}$ Tris p H 9) a $-20{ }^{\circ} \mathrm{C}$ hasta realizar la extracción de $\mathrm{ADN}$.

La extracción de ADN de los microorganismos recolectados sobre las membranas de $0,22 \mu \mathrm{m}$ se hizo siguiendo el método descrito por Castro-González, et al., (2005), consistente en digestión con lisozima $(1 \mathrm{mg} / \mathrm{ml})$ y proteinasa $\mathrm{K}(10 \mathrm{mg} /$ $\mathrm{ml})$, lisis celular por choque térmico, extracción con fenol, cloroformo y alcohol isoamílico $(25: 24: 1)$ y precipitación con etanol frío. La concentración y la pureza de las muestras se cuantificó por espectrofotometría a 260/280 nm utilizando un NanoDrop ${ }^{\text {TM }} 2000$.

La amplificación del gen funcional de la reductasa de óxido nitroso (nosZ) se hizo usando el par de cebadores nosZ1188FnosZ1869R descritos por Kloos, et al., (2001) para generar un amplicon de $700 \mathrm{pb}$. El cebador nosZ1188F se marcó con 6-carboxifluoresceina. La mezcla de reacción $(25 \mu \mathrm{l})$ incluyó $1 \mu \mathrm{l}$ de $\mathrm{ADN}(10 \mathrm{ng} / \mu \mathrm{l}), 2,5 \mu \mathrm{l}$ de cada cebador (10 $\mu \mathrm{M}), 0,5 \mu \mathrm{l}$ de polimerasa REDAccuTaq ${ }^{\circledR}$ (Sigma), 2,5 $\mu 1$ del tampón de reacción de la enzima, $0,5 \mu \mathrm{l}$ de la mezcla de dNTP $(200 \mu \mathrm{M})$ y $2,5 \mu \mathrm{l}$ de albumina de suero bovino $(4 \mu \mathrm{g} / \mathrm{ml})$ (Fermentas). La amplificación se estandarizó bajo las siguientes condiciones: desnaturalización a $94{ }^{\circ} \mathrm{C}$ durante 1 minuto, alineamiento durante los primeros 10 ciclos con touchdown de $0,5{ }^{\circ} \mathrm{C}$ comenzando a $57{ }^{\circ} \mathrm{C}$ y manteniendo los restantes 25 ciclos a $56{ }^{\circ} \mathrm{C}$, y una extensión final de 1 minuto a $72{ }^{\circ} \mathrm{C}$. Los productos de amplificación se tiñeron con bromuro de etidio $(0,5 \mathrm{mg} / \mathrm{l})$ y se analizaron por electroforesis en geles de agarosa al $2 \%$. Los productos de tres réplicas de PCR se combinaron y posteriormente se eluyeron del gel usando el estuche Wizard ${ }^{\mathrm{S} S V}$ Gel and PCR CleanUp System (Promega, Mannheim, Germany).
Los amplicones del nosZ purificados (100 ng) se digirieron con 5U de las enzimas HhaI y MspI en sus respectivos tampones de reacción siguiendo las instrucciones del proveedor, y los productos digeridos se limpiaron en columnas Autoseq G-50 (Amersham Biosciences), ajustándose a las instrucciones del proveedor. Se mezclaron alícuotas de 2 $\mu \mathrm{l}$ del producto digerido con $12 \mu \mathrm{l}$ de formamida desionizada (Applera, Darmstadt, Germany) y 0,2 $\mu 1$ del ADN de peso molecular estándar interno (X-Rhodamine map MarkerR 50-1000 bp; BioVentures, Murfreesboro, TN). Los fragmentos de restricción terminal (FRT) se separaron en un secuenciador ABI 310 (Applied Biosystems). Este análisis se hizo por triplicado para cada muestra con el fin de verificar la reproducibilidad de los FRT detectados, así como la fluorescencia relativa de cada uno de acuerdo a lo sugerido por Dunbar, et al., (2001). El largo (pb) de los FRT marcados con fluorescencia se determinó por comparación con el ADN estándar interno usando el programa Gene Scan 3.71 (Applied Biosystems).

Para el análisis de los FRT se escogieron los picos que presentaron $>50$ unidades de fluorescencia y $>45 \mathrm{pb}$. Los patrones obtenidos en cada profundidad se normalizaron en las mismas unidades de fluorescencia total usando el procedimiento de estandarización iterativa de Dunbar, et al., (2001). El porcentaje de abundancia relativa de cada FRT se determinó calculando la proporción entre la altura del pico dado y la altura total de los picos normalizados en cada profundidad.

Para el análisis de los datos se estimó el índice de diversidad de Shannon y Simpson, así como el índice de similitud de Bray Curtis, usando el programa Biodiversity Pro 2.0 (McAleece, 1997).

\section{Resultados y discusión}

\section{Parámetros fisicoquímicos}

El área de Isla del Sol se caracterizó por presentar condiciones óxicas $\left(4,1 \mathrm{mg} / 1\right.$ de $\left.\mathrm{O}_{2}\right)$ a 2 metros de profundidad e hipoxia $\left(1,1 \mathrm{mg} / \mathrm{l} \mathrm{de} \mathrm{O}_{2}\right)$ a $9 \mathrm{~m}$. La temperatura presentó una variación de $4{ }^{\circ} \mathrm{C}$ entre los 2 y los 9 metros. El nivel de nitrato y de nitrógeno amoniacal incrementó hacia los 9 metros de profundidad, mientras que el $\mathrm{pH}$ disminuyó y el nivel de nitrito fue muy bajo y cercano al límite de detección en ambas profundidades (Tabla 1)

Los datos de nutrientes, $\mathrm{pH}$ y temperatura superficiales concuerdan con los de estudios previos que han reportado un alto nivel de nitrógeno amoniacal $(0,316 \mathrm{mg} / \mathrm{l})$, y bajos niveles de nitratos $(0,206 \mathrm{mg} / \mathrm{l})$ y nitritos $(0,003 \mathrm{mg} / \mathrm{l})$, así como temperaturas altas y pH neutro en el embalse (Márquez \& Guillot, 1988; Canosa \& Pinilla, 2007). Dichos estudios han reportado también niveles elevados de sólidos suspendidos $(20,7 \mathrm{mg} / \mathrm{l})$ y de nitrógeno total $(7,8 \mathrm{mg} / \mathrm{l})$, además de bajos niveles de conductividad $(53,2 \mu \mathrm{S} / \mathrm{cm})$ y de alcalinidad $(13,0)$. 
Tabla 1. Parámetros ambientales y concentración de ADN obtenidos en cada profundidad de muestreo en la columna de agua en la estación de Isla del Sol del embalse de Prado

\begin{tabular}{cccccccc}
\hline $\begin{array}{c}\text { Profundidad } \\
(\mathbf{m})\end{array}$ & $\mathbf{O}_{\mathbf{2}}(\mathbf{m g} / \mathbf{L})$ & Temperatura ${ }^{\circ} \mathbf{C}$ & $\mathbf{p H}$ & $\begin{array}{c}\text { Nitrógeno amoniacal } \\
(\mathbf{m g} / \mathbf{L})\end{array}$ & $\begin{array}{c}\mathbf{N O}_{3}^{-} \\
(\mathbf{m g} / \mathbf{L})\end{array}$ & $\begin{array}{c}\mathbf{N O}_{2}^{-} \\
(\mathbf{m g} / \mathbf{L})\end{array}$ & $\begin{array}{c}\mathbf{A D N}^{-} \\
(\mathbf{n g} / \mathbf{\mu l})\end{array}$ \\
\hline 2 & 4,1 & 30,3 & 7,5 & 0,41 & 0,23 & $<0,05$ & 398,4 \\
9 & 1,1 & 26,4 & 6,2 & 0,59 & 0,78 & $<0,05$ & 359,5 \\
\hline
\end{tabular}

Por otra parte, el fuerte cambio fisicoquímico observado a los $9 \mathrm{~m}$ concuerda con los datos de estudios previos que indican la estratificación térmica y química del embalse con una fuerte oxiclina entre superficie y fondo, lo que genera anoxia por debajo de los $8 \mathrm{~m}$ en la mayor parte de la hidroeléctrica (Márquez \& Guillot, 1988). Estos datos complementan la información reportada en el área por la empresa Sodeic Ltda. (1993) y sugieren que las condiciones de estabilidad de la columna de agua y los procesos biológicos probablemente han llevado a la disminución de los niveles de oxígeno disuelto, creando condiciones hipóxicas, subóxicas y anóxicas a lo largo de la columna de agua que favorecen la presencia de los microorganismos desnitrificantes.

\section{Análisis molecular}

A partir del ADN obtenido se amplificó por PCR específicamente el gen funcional nos $Z$ con un amplicon de 700 pb. Al cortar el amplicon del gen nosZ de la comunidad desnitrificante se obtuvieron 15 fragmentos de restricción terminal diferentes con la enzima MspI (Figura 1) y 17 con la enzima HhaI (Figura 2), lo que indica su riqueza en la columna de agua. La riqueza de los FRT fue igual a los 2 y los $9 \mathrm{~m}$ de profundidad con la enzima MspI, en tanto que con la enzima HhaI se observó una mayor riqueza de FRT (16) a los $9 \mathrm{~m}$ de profundidad (Tabla 2). Se observó que la comunidad desnitrificante de tipo nosZ digerida con HhaI fue más homogénea en su estructura a los 2 y a $\operatorname{los} 9 \mathrm{~m}$, ya que la mayoría de los FRT observados a los $2 \mathrm{~m}$ (46, $69,82,91,103,146,288,319,405,474)$ también estaban presentes a los $9 \mathrm{~m}$ y con la misma abundancia relativa; sin embargo, los FRT únicos $(119,126,216,257,306,322)$, poco abundantes, se encontraron solamente a los $9 \mathrm{~m}$ de profundidad. En contraste, la estructura de la comunidad desnitrificante nosZ digerida con MspI se mostró un poco más heterogénea al comparar ambas profundidades, ya que hubo una menor abundancia de las diferentes especies comunes representadas en los FRT 55, 87, 102, 107, 110, 223 (con excepción de los FRT 130 y 140, los cuales incrementaron) a los $9 \mathrm{~m}$ de profundidad. De igual manera, con la enzima MspI se detectaron más FRT únicos tanto a los $2 \mathrm{~m}$ (76, 204, 223, 381) como a los $9 \mathrm{~m}(48,92,170,306)$ de profundidad, lo que sugiere que con esta enzima se obtuvo una mejor resolución de la riqueza de FRT de la comunidad desnitrificante de tipo nosZ en Isla del Sol.

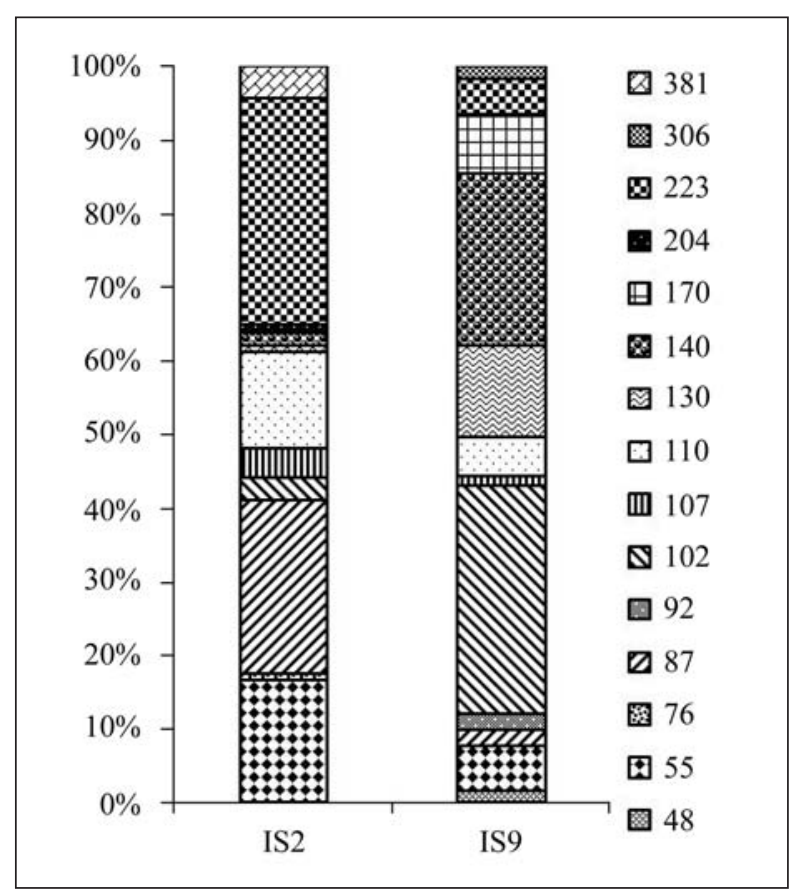

Figura 1. Abundancia relativa de los fragmentos de restricción terminal obtenidos con la enzima de restricción MspI representativos de la comunidad desnitrificante de tipo nosZ encontrados en Isla del Sol, embalse de Prado, a 2 m (IS2) y 9 m de profundidad (IS9). Los números en la leyenda indican el tamaño de cada fragmento (pares de bases).

Aunque se ha reportado que una tercera parte de las bacterias desnitrificantes presentes en el ambiente están genéticamente inhabilitadas para reducir el $\mathrm{N}_{2} \mathrm{O}$, dado que carecen del gen nosZ (Jones, et al., 2008), los resultados del presente trabajo demuestran que en el área de Isla del Sol del embalse de Prado existe una comunidad desnitrificante rica y diversa que posee el gen nos $Z$, el cual codifica para la reducción de óxido nitroso $\left(\mathrm{N}_{2} \mathrm{O}\right)$ a $\mathrm{N}_{2}$ tanto en condiciones hipóxicas en el fondo como en la superficie bajo condiciones óxicas, como en el fondo. Los resultados muestran que la riqueza de los FRT hallados en la columna de agua de Isla del Sol es similar a la reportada en sedimentos de arroyos (Baxter, et al., 2012) y en estuarios (Fortunato, et al., 2009), donde se han observado cambios espacio-temporales en la riqueza y en la abundancia relativa de FRT, con la diferencia de que en 


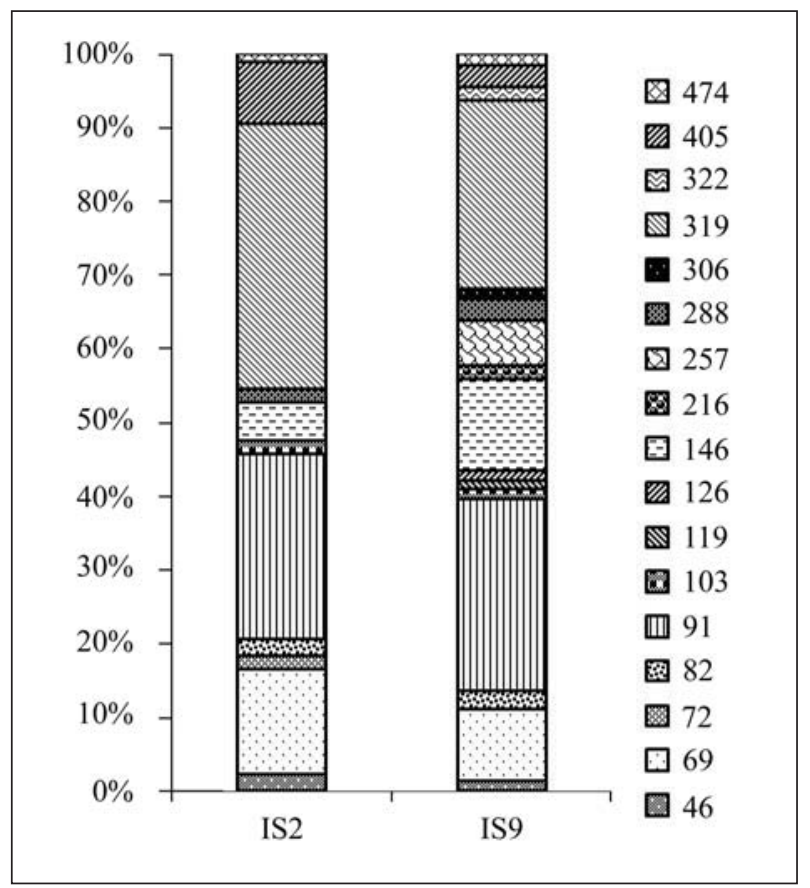

Figura 2. Abundancia relativa de los fragmentos de restricción terminal obtenidos con la enzima de restricción HhaI, representativos de la comunidad desnitrificante de tipo nosZ encontrados en Isla del Sol, embalse de Prado, a 2 m (IS2) y 9 m (IS9) de profundidad. Los números en la leyenda indican el tamaño de cada fragmento (pares de bases).

Tabla 2. Índices de diversidad, similitud y número de fragmentos de restricción terminal (FRT) obtenidos con las enzimas de restricción MspI y HhaI de las comunidades desnitrificantes de tipo nosZ presentes a $2 \mathrm{~m}$ (IS2) y $9 \mathrm{~m}$ (IS9) de profundidad en la columna de agua de Isla del Sol, embalse de Prado

\begin{tabular}{lcccc}
\hline & \multicolumn{2}{c}{ Enzima MspI } & \multicolumn{2}{c}{ Enzima HhaI } \\
\hline Índice de diversidad & IS2 & IS9 & IS2 & IS9 \\
\hline Shannon H' & 0,80 & 0,87 & 0,78 & 0,93 \\
Simpson 1/D & 5,0 & 5,5 & 4,5 & 6,0 \\
\hline Índice de similitud de Bray Curtis & $24 \%$ & - & $77 \%$ & - \\
Número de FRT & 11 & 12 & 11 & 16 \\
\hline
\end{tabular}

dichos sedimentos las concentraciones de nutrientes, nitrógeno total y materia orgánica tienden a ser mayores que las que usualmente se encuentran en la columna de agua.

Los resultados de esta investigación respaldan lo verificado en otros estudios en el sentido de que herramientas moleculares como los FRT, la clonación y, más recientemente, la pirosecuenciación (Hallin, et al., 2007; Magalhaes, et al., 2008; Fortunato, et al., 2009; Bai, et al., 2012; Eiler, et al., 2012; Wang, et al., 2012; Baxter, et al., 2012; Jones, et al., 2013; Bowen, et al., 2013), han ayudado a develar la diversidad de los microorganismos desnitrificantes en los ecosistemas acuáticos de agua dulce, aunque esta siga siendo mucho menor que la encontrada en los ecosistemas terrestres (Braker \& Conrad, 2011). Sin embargo, se debe tener presente que aunque la técnica de FRT se ha usado ampliamente para el estudio de comunidades microbianas, pues permite obtener un perfil de las comunidades de manera reproducible y exacta, también está sujeta a las limitaciones propias de la amplificación por PCR (la concentración del segmento de ADN, el número de ciclos de la PCR, la amplificación preferencial de segmentos de ADN específico de acuerdo a los cebadores usados), y a errores en el proceso de digestión con enzimas (debidos a una digestión incompleta o a la presencia de diferentes sitios de restricción terminal en la misma especie como resultado de las múltiples copias del gen en estudio), como lo han sugerido Egert \& Friedrich (2003), lo que puede impedir la detección de especies con muy poca abundancia o que poseen secuencias características, o en el caso contrario, conducir a la sobreestimación de la diversidad por la presencia de fragmentos de restricción terminal falsos y de pseudo fragmentos, si no se analizan correctamente los datos.

\section{Análisis de diversidad y similitud}

Los índices de diversidad de Shannon $\left(\mathrm{H}^{\prime}\right)$ y Simpson (1/D) (Tabla 2) mostraron que la comunidad digerida con MspI y HhaI a los $9 \mathrm{~m}$ era un poco más diversa $\left(\mathrm{H}^{\prime} \sim 0,9\right)$ que la comunidad a los $2 \mathrm{~m}$ de profundidad $\left(\mathrm{H}^{\prime} \sim 0,8\right)$. También se observó que la similitud de las comunidades desnitrificantes de tipo nosZ entre los 2 y $\operatorname{los} 9 \mathrm{~m}$ fue de un $77 \%$ al analizarlas mediante la digestión con HhaI y de solo $24 \%$ al analizar los FRT con MspI (Tabla 2). Estos resultados revelaron diferencias en la composición de las comunidades de tipo nosZ entre las dos profundidades y evidenciaron que la diversidad de la comunidad de tipo nosZ encontrada en la columna de agua de Isla del Sol es menor que la reportada para sedimentos de estuarios $\left(\mathrm{H}^{\prime}=1,5-2,6\right)$ y sedimentos de lagos eutróficos $\left(\mathrm{H}^{\prime}=2,5\right)$ por Fortunato, et al., (2009) y Wang, et al., (2012a). Estas diferencias son lógicas teniendo en cuenta que los sedimentos usualmente poseen mayor cantidad de materia orgánica y de nutrientes que la columna de agua. Sin embargo, y habida cuenta de que no hay estudios similares en embalses para poder comparar, sí se debe tener en cuenta que se ha reportado mayor diversidad de bacterias y arqueobacterias en la represa de Three Gorges en China en dos casos: el primero, cuando los niveles de nutrientes, de carbono orgánico disuelto y de turbidez eran altos, y el segundo, cuando se estudiaron las bacterias asociadas a la fracción particulada (Wang, et al., 2012). Además, otros autores también han hecho referencia al hecho de que pueden darse variaciones en la estructura de las comunidades de tipo nosZ debidas a su interacción con las bacterias nitrificantes 
(Deemer, et al., 2011) y las bacterias anammox (Wenk, et al., 2013), así como a los factores que regulan la expresión del gen nosZ y la actividad de su reductasa (Baxter, et al., 2012).

Por otra parte, los resultados de similitud entre comunidades sugieren que el análisis de FRT realizado con la enzima MspI permitió una mejor resolución de la comunidad de tipo nosZ en ambas profundidades, lo que se reflejó en una baja similitud entre las comunidades a los 2 y $\operatorname{los} 9 \mathrm{~m} \mathrm{de}$ profundidad $(24 \%)$ y la presencia de mayor número de FRT únicos que los observados con la enzima HhaI. Estos datos concuerdan con lo reportado en varios estudios en los cuales se hace referencia a que la selección adecuada de la enzima para generar los FRT, así como del procedimiento de análisis de los FRT, es de suma importancia en el análisis de la composición de las comunidades microbianas (Dunbar, et al., 2001).

\section{Composición de la comunidad de tipo nosZ y su relación con el ambiente}

El análisis de los FRT mostró una mayor diversidad de la comunidad de tipo nosZ en Isla del Sol a los $9 \mathrm{~m}$ de profundidad, donde probablemente se registra una mayor tasa de reducción de $\mathrm{N}_{2} \mathrm{O}$ a $\mathrm{N}_{2}$ debido a las condiciones hipóxicas $\left(1,1 \mathrm{mg} / \mathrm{l}\right.$ de $\left.\mathrm{O}_{2}\right)$, de acidez $(\mathrm{pH}=6,2)$ y de disponibilidad de nitrato $(0,78 \mathrm{mg} / \mathrm{l})$ y de $\mathrm{N}_{2} \mathrm{O}(\sim 40 \mathrm{nM})$, según recientes cuantificaciones a esta profundidad (Castro-González \& Torres-Valdés, 2014). Asimismo, en el embalse se observó que las bacterias desnitrificantes de tipo nos- $Z$ se encuentran en la columna de agua a partir de los $2 \mathrm{~m}$ de profundidad bajo condiciones óxicas $\left(4 \mathrm{mg} / \mathrm{l}\right.$ de $\left.\mathrm{O}_{2}\right)$, neutras $(\mathrm{pH}=7,5)$ y de bajo nitrato $(0,23 \mathrm{mg} / \mathrm{l})$, lo que sugiere su versatilidad metabólica ante diferentes concentraciones de $\mathrm{O}_{2}, \mathrm{pH}$ y nutrientes; sin embargo, se requieren más estudios que permitan determinar si la óxido nitroso reductasa es activa bajo estas condiciones óxicas o está inhibida. En general, son varios los estudios que al igual que este muestran cómo los filotipos, las unidades taxonómicas operacionales (UTO) o los FRT de comunidades desnitrificantes de tipo nirS, nirK y nosZ están presentes a lo largo de gradientes fisicoquímicos en la columna de agua de océanos (Castro-González, et al., 2005; Qian, et al., 2011; Ganesh, et al., 2013) y de lagos (Wen, et al., 2012), así como en sedimentos tanto de estuarios (Abell, et al., 2010,) como de lagos (Bai, et al., 2012; Wang, et al., 2012a;), lo que indica la capacidad facultativa de las bacterias desnitrificantes en ambientes con alta variabilidad ambiental. En este sentido, Magalhaes, et al. (2008) reportaron variación en la abundancia relativa de FRT tipo nosZ con relación a la disponibilidad de nitrato y las tasas de desnitrificación en sedimentos; Bai, et al. (2012) reportaron cambios espacio-temporales en la abundancia relativa de los FRT y en la abundancia de genes nosZ detectada por PCR cuantitativa en sedimentos del lago Dianchi; Baxter, et al. (2013) reportaron cambios en la abundancia de genes nosZ relacionados con la temperatura y el porcentaje de materia orgánica del sedimento en diferentes arroyos, y CastroGonzález, et al. (2005) reportaron una mayor riqueza de FRT y mayor diversidad de las comunidades desnitrificantes (nirS) asociadas a la variación en los niveles de oxígeno, nitrito y óxido nitroso en aguas oceánicas subóxicas.

Si bien los resultados de este estudio indican que hay comunidades desnitrificantes que poseen el gen nos $Z$ y que su abundancia relativa, riqueza y diversidad está relacionada con algunas de las características estudiadas en la columna de agua, el siguiente paso en la investigación deberá orientarse a determinar aspectos como la variación espacio-temporal de esta comunidad y de las comunidades desnitrificantes de tipo nirS, nirK, y la comunidad anammox, la cual también ha sido reportada en lagos (Schubert, et al., 2006; Penton, et al., 2006; Wenk, et al., 2013) y que, por consiguiente, puede estar presente en el embalse y contribuir a la generación de $\mathrm{N}_{2}$. Además, habría que determinar la funcionalidad de la $\mathrm{N}_{2} \mathrm{O}$ reductasa en el área, teniendo en cuenta que: 1) esta es inhibida reversiblemente por los niveles elevados de oxígeno; 2) algunos microorganismos desnitrificadores, aunque poseen el gen nosZ, no reducen el $\mathrm{N}_{2} \mathrm{O}$ a $\mathrm{N}_{2}$ (Wang, et al., 2012a); 3 ) se ha reportado una relación negativa entre la abundancia de genes nos $\mathrm{Z}$ y la proporción de $\mathrm{N}_{2} \mathrm{O}$ que se reduce a $\mathrm{N}_{2}$ en sedimentos de lagos eutróficos (Wang, et al., 2012a), y 4) en recientes trabajos se ha cuantificado una saturación excesiva de $\mathrm{N}_{2} \mathrm{O}(\sim 200 \%)$ en la columna de agua del embalse, lo cual sugiere un eflujo de este gas invernadero hacia la atmósfera (Castro-González \& Torres-Valdes, 2014).

\section{Conclusiones}

Este es el primer trabajo de investigación en Colombia en que se demuestra la existencia de comunidades desnitrificantes de tipo nos $Z$ en la estación de Isla del Sol en el embalse de Prado mediante el estudio molecular de su gen funcional. El análisis de la composición de dicha comunidad en la columna de agua de esta estación mostró una alta diversidad de FRT en comparación con la reportada en otros ambientes acuáticos, además de cambios en su composición (abundancia relativa y riqueza) entre la superficie y el fondo. La mayor diversidad de la comunidad y el mayor número de FRT únicos se detectaron a los $9 \mathrm{~m}$ de profundidad bajo condiciones hipóxicas, punto en el que probablemente se requiere una mayor tasa de reducción de $\mathrm{N}_{2} \mathrm{O}$ a $\mathrm{N}_{2}$ para favorecer el proceso de la desnitrificación.

\section{Conflicto de intereses}

La autora declara que no tiene ningún conflicto de intereses.

\section{Agradecimientos}

A la oficina de investigaciones y desarrollo científico de la Universidad del Tolima por la financiación de esta investigación a través del proyecto \# 500110, al Ministerio del 
Medio Ambiente que, a través del "Contrato de acceso a recursos genéticos y productos derivados para investigación científica sin interés comercial No. 90", autorizó el acceso y el uso del ADN bacteriano ambiental requerido para este estudio, y a A. Castro y M. Pacheco por su apoyo en campo $\mathrm{y}$ en el laboratorio.

\section{Bibliografía}

Abell, G.C.J, Revill, A.T., Smith, C., Bissett, A.P., Volkman, J.K., Stanley, S.R. 2010. Archaeal ammonia oxidizers and nirS-type denitrifiers dominate sediment nitrifying and denitrifying populations in a subtropical macrotidal estuary. ISME Journal 4: 286-300.

APHA. 1999. Standard methods for the examination of water and wastewater. Twentieth edition. Baltimore, Maryland. 1220 pp.

Bai, Y., Shi, Q., Wen, D., Li, Z., Jefferson, W.A., Feng, Ch., Tang, X. 2012. Bacterial communities in the sediments of Dianchi Lake, a partitioned eutrophic water body in China. PLoS ONE 7 (5): e37796.

Baxter, A.M., Johnson, L., Royer, T., Leff, L.G. 2013. Spatial differences in denitrification and bacterial community structure of streams: Relationships with environmental conditions. Aquatic Science 75: 275-284.

Baxter, A.M., Johnson, L., Edgerton, J., Royer, T., Left, L.G. 2012. Structure and function of denitrifying bacterial assemblages in low-order Indiana streams. Freshwater Science 31 (2): $304-317$

Beaulieu, J.J., Smolensky, R.L., Nietch, C.T., TownsendSmall, A., Elovitz, M.S., Schubauer-Berigan, J.P. 2014. Denitrification alternates between a source and sink of nitrous oxide in the hypolimnion of a thermally stratified reservoir. Limnology and Oceanography 59 (2): 495-506.

Bowen, J.L., Bymes, J.E.K., Wisman, D., Colaneri, C. 2013. Functional gene pyrosequencing and network analysis: An approach to examine the response of denitrifying bacteria to increase nitrogen supply in salt marsh sediments. Frontiers in Microbiology 4: 1-12.

Braker, G. \& Conrad, R. 2011. Diversity, structure, and size of $\mathrm{N}_{2} \mathrm{O}$ producing microbial communities in soils. What matters for their functioning? Advances in Applied Microbiology 75: $33-70$

Brettar, I. \& Höfle, M.G. 1993. Nitrous oxide producing heterotrophic bacteria from the water column of the central Baltic: Abundance and molecular identification. Marine Ecology Progress Series 94: 253-265.

Canosa, A. \& Pinilla, G. 2007. Relaciones entre las abundancias del bacterioplancton y del fitoplancton en tres ecosistemas lenticos de los Andes Colombianos. Revista de Biología Tropical 55 (1): 135-146.

Castro-González, M. \& Torres-Valdés, V. (2014). Microbial process involved in the distribution and $\mathrm{N}_{2} \mathrm{O}$ cycling in the water column in a tropical hydroelectric reservoir, south east Colombia. $15^{\text {th }}$ International Symposium on Microbial Ecology. ISME-Microbes.org. Seoul, South Korea.

Castro-González, M., Braker, G., Farías, L., Ulloa, O. 2005. Communities of nirS-type denitrifiers in the water column of the oxygen minimum zone in the eastern South Pacific. Environmental Microbiology 7 (9): 1298-1306.

Deemer, B.R., Harrison, J.A., \& Whitling, E.W. 2011. Microbial dinitrogen and nitrous oxide production in a small eutrophic reservoir: An in situ approach to quantifying hypolimnetic process rates. Limnology and oceanography 56 (4): 11891199.

Dong, L.F., Smith, C.J., Papaspyrou, S., Stott, A., Osborn, A.M., Nedwell, D.B. 2009. Changes in benthic denitrification, nitrate ammonification and anammox process rates and nitrate and nitrite reductase gene abundances along an estuarine nutrient gradient (The Colne Estuary, United Kingdom). Applied and Environmental Microbiology 75 (10): 3171-3179.

Dunbar, J., Ticknor, L.O. \& Kuske, C.H.R. 2001. Phylogenetic specificity and reproducibility and new method for analysis of terminal restriction fragment profiles of 16S rRNA genes from bacterial communities. Applied and Environmental Microbiology 67 (1): 190-197.

Egert, M. \& Friedrich M.W. 2003. Formation of pseudo-terminal restriction fragments, a PCR-related bias affecting terminal restriction fragment length polymorphism analysis of microbial community structure. Applied and Environmental Microbiology 69 (5): 2555-2562.

Eiler, A., Heinrich, F. \& Bertilsson, S. 2012. Coherent dynamics and association networks among lake bacterioplankton taxa. The ISME Journal 6: 330-342.

Fortunato, C.S., Carlini, D.B., Ewers, E., Bushaw-Newton, K.L. 2009. Nitrifier and denitrifier molecular operational taxonomic unit compositions from sites of a freshwater estuary of Chesapeake Bay. Canadian Journal of Microbiology 55: 333-346.

Ganesh, S., Parris, D.J., Delong, E.F., Stewart, F.J. 2013 Metagenomic analysis of size-fractionated picoplankton in a marine oxygen minimum zone. The ISME Journal 1: 1-25.

Goldenfum, J.A. 2012. Challenges and solutions for assessing the impact of freshwater reservoir on natural GHG emissions. Ecohydrology and Hydrobiology 12 (2): 115-122.

Hallin, S., Braker, G. \& Philippot L. 2007. Molecular tools to assess the diversity and density of denitrifying bacteria in their habitats. In: Biology of the nitrogen cycle. Edited by H. Bothe, SJ Ferguson and WE Newton. Elsevier BV. 40 p.

Harrison, J.A., Maranger, R.J., Alexander, R.B., Giblin, A.E., Jacinthe, P-A., Mayorga, E., Seitzinger, S.P., Sobota, D.J., Wollheim, W.M. 2009. The regional and global significance of nitrogen removal in lakes and reservoirs. Biogeochemistry 93: 143-157.

Hulth, S., Allerb, R.C., Canfield, D.E., Dalsgaard, T., Engstrom, P., Gilberte, F., Sundback, K., Thamdrup, B.O. 2005. Nitrogen removal in marine environments: 
Recent findings and future research challenges. Marine Chemistry 94: 125-145.

Jones, C.M., Stres, B., Rosenquist, M., Hallin, S. 2008. Phylogenetic analysis of nitrite, nitric oxide, and nitrous oxide respiratory enzymes reveal a complex evolutionary history for denitrification. Molecular Biology and Evolution 25: 1955-1966.

Jones, C.M., Graf, D.R.H., Bru. D., Philippot, L., Hallin, S. 2013. The unaccounted yet abundant nitrous oxide-reducing microbial community: A potential nitrous oxide sink. The ISME Journal 7: 417-426.

Kloos, K., Mergel, A., Rosch, C., Bothe, H. 2001. Denitrification within the genus Azospirillum and other associative bacteria. Australian Journal Plant Physiology 28: 991-998.

Mc Aleece, N. 1997. BioDiversity Professional Beta 2.0. The Natural History Museum.

Magalhaes, C., Bano, N., Wiebe, W.J., Bordalo, A.A., Hollibaugh, J.T. 2008. Dynamics of nitrous oxide reductase genes (nosZ) in intertidal rocky biofilms and sediments of the Duoro River estuary (Portugal), and their relation to N-biogeochemistry. Microbial Ecology 55: 259-269.

Márquez, G. \& Guillot, G. 1988. Proyecto estudios ecológicos de embalses colombianos. Etapa Prospectiva, Informe Final, Fondo FEN. Universidad Nacional de Colombia. Bogotá, Colombia, $242 \mathrm{p}$.

Naqvi, S.W.A., Bange, H.W., Farías, L., Monteiro, P.M.S., Scranton, M.I., Zhang, J. 2010. Marine hypoxia/anoxia as a source of $\mathrm{CH}_{4}$ and $\mathrm{N}_{2} \mathrm{O}$. Biogeosciences 7: 2159-2190

Naqvi, S.W.A., Jayakumar, D.A., Narvekar, P.V., Naik, H., Sarma, V.V.S.S., D'Souza, W., Joseph, S., George, M.D. 2000. Increased marine production of $\mathrm{N}_{2} \mathrm{O}$ due to intensifying anoxia on the Indian continental shelf. Nature 408: $346-349$.

Palmer, K., Biasi, C. \& Horn, M.A. 2012. Contrasting denitrifier communities relate to contrasting $\mathrm{N}_{2} \mathrm{O}$ emission patterns from acidic peat soils in Arctic tundra. The ISME Journal 6: $1058-1077$.

Penton, C.R., Devol, A.H., Tiedje, J.M. 2006. Molecular evidence for the broad distribution of anaerobic ammonium-oxidizing bacteria in freshwater and marine sediments. Applied and environmental microbiology 72 (10): 6829-6832.

Qian, P.Y., Wang, Y., Lee, O.O., Lau, S.C.K., Yang, J., Lafi, F.F. 2011. Vertical stratification of microbial communities in the Red Sea revealed by $16 \mathrm{~S}$ rDNA pyrosequencing. The ISME Journal 5: 507-518.

Rudd, J.W.M., Harris, R., Kelly, C.A., Hecky, R.E. 1993. Are hydroelectric reservoirs significant sources of greenhouse gases? Ambio 22: 246-248.

Rusch, A. 2013. Molecular tools for the detection of nitrogen cycling Archaea. Archaea. Article ID 676450, 10 pages. doi:10.1155/2013/676450.
Seitzinger, S., Harrison, J.A., Böhlke, J.K., Bouwman, A.F., Lowrance, R., Peterson, B., Tobias, C., Van Dreche, G. 2006. Denitrification across landscapes and waterscapes: A synthesis. Ecology Applied 16: 2064-2090.

Schubert, C.J., Durish-Kaiser, E., Wehrli, B., Thamdrup, B., Lam, P., Kuypers, M.M.M. 2006. Anaerobic ammonium oxidation in a tropical freshwater system (Lake Tanganyika). Environmental Microbiology 8: 1857-1863.

Sodeic Ltda. 1993. Plan de manejo de la cuenca del río Prado. Informe Final de Consultoría. 3 Tomos.

St Louis, V., Kelly, L.C.A., Duchemin, E., Rudd, J.W.M., Rosenberg, D.M. 2000. Reservoir surfaces as sources of greenhouse gases to the atmosphere: A global estimate. BioScience 50: 766-775.

Taipale, S., Jones, R.I. \& Tiirola, M. 2009. Vertical diversity of bacteria in an oxygen-stratified humic lake, evaluated using DNA and phospholipid analyses. Aquatic Microbial Ecology 55: 1-16.

Throbäck, I.N., Enwall, K., Jarvis, A., Hallin, S. 2004 Reassessing PCR primers targeting nirS, nirK and nosZ genes for community surveys of denitrifying bacteria with DGGE. FEMS Microbiology and Ecology 49: 401-417.

Wang, S., Dong, R.M., Dong, C.Z., Huang, L., Jiang, H., Wei, Y., Feng, L., Liu, D., Yang, G., Zhang, Ch., Dong, H. 2012. Diversity of microbial plankton across the Three Gorges dam of the Yangtze River, China. Geoscience Frontiers 3 (3): 335-349.

Wang, C., Zhu, G., Wang, Y., Wang, S., Yin, Ch. 2012a. Nitrous oxide reductase gene (nosZ) and $\mathrm{N}_{2} \mathrm{O}$ reduction along the littoral gradient of a eutrophic freshwater lake. Journal of Environmental Sciences 25 (1): 44-52.

Wen, D.H., Bai, Y.H., Shi, Q., Li, Z.X., Sun, Q.H., Sun, R.H., Feng, C.P., Tang, X.Y. 2012. Bacterial diversity in the polluted water of the Dianchi Lakeshore in China. Annals of Microbiology 62 (2): 715-723.

Wenk, C.B., Blees, J., Zopfi, J., Veronesi, M., Bourbonnais, A., Schubert, C.J., Niemann, H., Lehmann, M.F. 2013. Anaerobic ammonium oxidation (anammox) bacteria and sulfide-dependent denitrifies coexist in the water column of a meromictic south-alpine lake. Limnology and Oceanography 58 (1): 2013, 1-12.

Zumft, W.G. 1997. Cell Biology and Molecular Basis of Denitrification. Microbiology and Molecular Biology Reviews 61 (4): 533-616.

Zumft, W. \& Kroneck, P. 2007. Respiratory transformation of nitrous oxide $\left(\mathrm{N}_{2} \mathrm{O}\right)$ to dinitrogen by Bacteria and Archaea. Advances in Microbiology and Physiology 52: 107-227. 